\title{
Self-oligomerization of the CARD domain prevents complex formation in the CARMA1 signalosome
}

\author{
JIN HEE PARK ${ }^{*}$, JU YOUNG BAE* and HYUN HO PARK \\ School of Biotechnology and the Graduate School of Biochemistry, \\ Yeungnam University, Gyeongsan, Republic of Korea
}

Received November 21, 2012; Accepted January 18, 2013

DOI: 10.3892/ijmm.2013.1307

\begin{abstract}
The CARMA1 signalosome composed of CARMA1, BCL10 and MALT1 plays a pivotal role in antigen receptormediated lymphocyte activation via the NF- $\mathrm{B}$ pathway. For assembly of the CARMA1 signalosome, BCL10 functions as an adaptor protein that interacts with CARMA1 via the CARDCARD interaction and with MALT1 via interaction between the C-terminal Ser/Thr-rich region of BCL10 and the first Ig domain of MALT1. Despite the biological importance of the CARMA1 signalosome, structural and biochemical studies have been limited as CARD-containing proteins are prone to aggregation under physiological conditions. In the present study, we successfully purified and characterized CARMA1 CARD and BCL10 CARD and showed that both CARMA1 CARD and BCL10 CARD easily self-oligomerized under physiological conditions. This self-oligomerization of the CARD domain prevents complex formation in the CARMA1 signalosome in vitro. Finally, we propose an interaction mode between CARMA1 CARD and BCL10 CARD based on a structure-based modeling study.
\end{abstract}

\section{Introduction}

The transcription factors $\mathrm{NF}-\kappa \mathrm{B}$ and Jun N-terminal kinase (JNK) are crucial for cell survival, development, lymphocyte activation and proliferation. Genetic deficiencies in NF- $\mathrm{B}$ or its signaling components that act upstream of $\mathrm{NF}-\kappa \mathrm{B}$ have been shown to cause immune deficiencies, whereas over-activation of $\mathrm{NF}-\kappa \mathrm{B}$ has been linked to autoimmunity and neoplastic disorders $(1,2)$. The CARMA1 signalosome, which is composed of CARMA1 [caspase recruitment domain (CARD)-containing MAGUK protein 1], BCL10 (B-cell

Correspondence to: Professor Hyun Ho Park, School of Biotechnology and Graduate School of Biochemistry, Yeungnam University, Dae-dong, Gyeongsan, Republic of Korea

E-mail: hyunho@ynu.ac.kr

*Contributed equally

Key words: $\mathrm{T}$ cell activation, inflammation, NF- $\mathrm{B}$, CARMA1 signalosome, CARD domain lymphoma 10) and MALT1 (mucosa-associated lymphoid tissue lymphoma translocation protein 1), is the main signaling molecular complex that performs pivotal functions in T-cell receptor (TCR)- and B-cell receptor (BCR)-mediated $\mathrm{NF}-\kappa \mathrm{B}$ activation.

CARMA1 (also called CARD11) is a member of the MAGUK (membrane-associated guanylate kinase) family of scaffold proteins that assists in recruitment and assembly of signaling molecules in the cytoplasmic membrane. CARMA1 contains an N-terminal CARD domain followed by a coiledcoil domain and a C-terminal MAGUK domain $(3,4)$. The CARD domain is a subfamily of the death domain (DD) superfamily that comprises the DD subfamily, death effector domain (DED) subfamily, caspase recruitment domain (CARD) subfamily and pyrin domain (PYD) subfamily. The death domain superfamily, which is one of the largest classes of protein interaction modules, plays a role in apoptosis, inflammation, and innate immune signaling pathways (5-8). MALT1 contains an N-terminal DD followed by two immunoglobulin (IG)-like domains and a C-terminal caspase-like domain. Although the molecular mechanism of MALT1 involvement in TCR- or BCR-mediated NA- $\kappa \mathrm{B}$ activation is unclear, several studies have shown that MALT1 functions as an ubiquitin E3 ligase inducing K63-linked polyubiquitination of NEMO, which is one of the components of the IкB kinase (IKK) complex, and that it also functions as a protease that can cleave the deubiquitinating enzymes A20 and BCL10 (9-12). The proteolytic activity of MALT1 is required for proper $\mathrm{NF}-\kappa \mathrm{B}$ activation followed by IL-2 production $(11,12)$. BCL10 is composed of an N-terminal CARD and a C-terminal Ser/ Thr-rich region and has been shown to induce apoptosis and activate NF- $\kappa$ B. For assembly of the CARMA1 signalosome, BCL10 functions as an adaptor protein that interacts with CARMA1 via the CARD-CARD interaction and with MALT1 via interaction between the C-terminal Ser/Thr-rich region of BCL10 and the first Ig domain of MALT1 $(13,14)$. Upon the activation of $\mathrm{T}$ cells, TCR transmits an outside signal to PKC $\theta$ that can then phosphorylate downstream target CARMA1 $(15,16)$. This phosphorylation promotes the assembly of the CARMA1 signalosome (16).

Due to the importance of the CARMA1 signalosome in immune receptor signaling events, studies in these fields are of great biological importance. Despite the biological importance of the CARMA1 signalosome, structural and biochemical 
studies have been limited as CARD-containing proteins are prone to aggregation under physiological conditions (17-19). In the present study, we successfully purified and characterized CARMA1 CARD and BCL10 CARD and showed that both CARMA1 CARD and BCL10 CARD easily self-oligomerized under physiological conditions. This self-oligomerization of the CARD domain prevents complex formation in the CARMA1 signalosome in vitro. Finally, we proposed an interaction mode between CARMA1 CARD and BCL10 CARD based on a structural-based modeling study.

\section{Materials and methods}

Materials. DNA restriction enzymes (NdeI and XhoI), T4 DNA ligase, and Tag DNA polymerase were obtained from NEB (New England Biolabs, Ipswich, MA, USA). IPTG, urea, imidazole and DTT were purchased from Sigma (St. Louis, MO, USA). E. coli BL21 (DE3) cells and plasmid pET vectors were obtained from Novagen. A Superdex 200 gel filtration column was purchased from GE Healthcare. Inhibitor cocktail was acquired from Roche Diagnostics (Indianapolis, IN, USA). All concentrators were obtained from Millipore (Billerica, MA, USA).

Expression of CARMA1 CARD and BCL1O CARD in E. coli. The cDNA of full length mouse CARMA1 (1-1155 amino acids) and BCL10 (1-233 amino acids) were used as templates for PCR, while plasmid vectors pET26b and pET28a were used to add a hexahistidine (6-His) tag to the C-terminus and the $\mathrm{N}$ and C-terminus, respectively, for affinity purification. The CARD domain of mouse CARMA1 coding for 1(Met)-109(Lys) was cloned into pET 28a, resulting in pET (6His-CARMA1 CARD-6His). Additionally, the CARD domain of BCL10 coding for 1(Met)-93(Asn) was cloned into pET 26b, resulting in pET (BCL10 CARD-6His). The expression methods for two proteins were the same as previously described (20). A brief summary of these methods is presented below.

Each clone was streaked onto Lysogeny broth (LB) agar plates containing appropriate antibiotics $(100 \mu \mathrm{l} / \mathrm{ml}$ ampicillin for the pET $26 \mathrm{~b}$ clone and $50 \mu \mathrm{l} / \mathrm{ml}$ kanamycin for the $\mathrm{pET}$ 28a clone), after which the plates were incubated at $37^{\circ} \mathrm{C}$ for $18 \mathrm{~h}$. A single colony was then used to inoculate $5 \mathrm{ml}$ of the LB medium, which was subsequently incubated at $37^{\circ} \mathrm{C}$ in a shaking incubator. Next, $5 \mathrm{ml}$ of the overnight small-scale culture was used to inoculate $1000 \mathrm{ml}$ of LB in a 2000-ml culture flask, and the culture growth was continued in a shaking incubator for $\sim 4 \mathrm{~h}$. When the optical density (OD) at $600 \mathrm{~nm}$ reached 0.6-0.7, expression was induced by overnight treatment with $0.5 \mathrm{mM}$ isopropyl- $\beta$-D thiogalactopyranoside (IPTG) at $20^{\circ} \mathrm{C}$.

Purification of target proteins. Bacteria expressing each protein were pelleted by centrifugation, resuspended and lysed by sonication in $50 \mathrm{ml}$ of lysis buffer [ $20 \mathrm{mM}$ sodium citrate (pH 5.0), $500 \mathrm{mM} \mathrm{NaCl}$ and $5 \mathrm{mM}$ imidazole] supplied with a protease inhibitor-cocktail (Roche Diagnostics). The lysate was then centrifuged at $16,000 \mathrm{rpm}$ for $1 \mathrm{~h}$ at $4^{\circ} \mathrm{C}$, after which the supernatant fractions were applied to a gravity column (Bio-Rad, Hercules, CA, USA) packed with Ni-NTA affinity resin (Qiagen, Hilden, Germany). Next, the unbound bacterial proteins were removed by rinsing with $100 \mathrm{ml}$ of washing buffer (20 mM Tris buffer at $\mathrm{pH} 8.0,500 \mathrm{mM} \mathrm{NaCl}$, $60 \mathrm{mM}$ imidazole), after which the target proteins were eluted from the column using elution buffer $[20 \mathrm{mM}$ sodium citrate (pH 5.0), $500 \mathrm{mM} \mathrm{NaCl}$ and $250 \mathrm{mM}$ imidazole], with $1 \mathrm{ml}$ elution fractions being collected over a total of $10 \mathrm{ml}$. Fractions containing $>80 \%$ homogeneous target protein as shown by SDS-PAGE were then selected and combined. In the final purification step, the sample was loaded onto a Superdex-200 gel-filtration column (GE Healthcare, Sweden) equilibrated with various buffers. Fractions containing the target protein were then pooled and stored at $4^{\circ} \mathrm{C}$ for further experiments. The homogeneity of the protein was assessed by SDS-PAGE.

Circular dichroism spectroscopy. The secondary structures were measured by circular dichroism (CD) spectroscopy using a J-715 spectropolarimeter (Jasco, Japan). The spectra were obtained from 200 to $250 \mathrm{~nm}$ at $25^{\circ} \mathrm{C}$ in a $0.1-\mathrm{cm}$ pathlength quartz cuvette using a bandwidth of $1.0 \mathrm{~nm}$, a speed of $50 \mathrm{~mm} / \mathrm{min}$, and a 5 -sec response time. The protein samples in the buffer containing $20 \mathrm{mM}$ Tris- $\mathrm{HCl}$ at $\mathrm{pH} 8.0$ and $150 \mathrm{mM}$ $\mathrm{NaCl}$ were diluted to $0.1 \mathrm{mg} / \mathrm{ml}$ prior to use. Four scans were accumulated and averaged and the $\alpha$-helical content was calculated from the molar ellipticity at $222 \mathrm{~nm}(21)$.

Complex assay by gel-filtration chromatography. Purified CARMA1 was incubated with BCL10 for $1 \mathrm{~h}$ at room temperature. Following incubation, the mixture was concentrated to $10-15 \mathrm{mg} \mathrm{ml}^{-1}$ using a concentration kit (Millipore). The concentrated protein mixture was then applied to a Superdex 200 gel-filtration column 10/30 (GE Healthcare), which was pre-equilibrated with a solution of $20 \mathrm{mM}$ Tris buffer at $\mathrm{pH} 8.0$ and $0 \mathrm{mM} \mathrm{NaCl} ; 20 \mathrm{mM}$ Tris buffer at $\mathrm{pH} 8.0$ and $150 \mathrm{mM} \mathrm{NaCl}$; and $20 \mathrm{mM}$ Tris buffer at $\mathrm{pH} 8.0$ and $1 \mathrm{M}$ $\mathrm{NaCl}$. Assembly of the complex was evaluated based on the positions of the eluted peaks monitored at $280 \mathrm{~nm}$ followed by SDS-PAGE.

Native-PAGE assay. Formation of the complex between CARMA1 CARD and BCL10 CARD was assayed by native (non-denaturing) PAGE conducted on a PhastSystem (GE Healthcare) with pre-made $8-25 \%$ acrylamide gradient gels (GE Healthcare). Coomassie Brilliant Blue was used for staining and detection of the band patterns. Separately purified CARMA1 CARD and BCL10 CARD were pre-incubated for $1 \mathrm{~h}$ at room temperature, after which the mixture was applied to the gel. The assembly of the complex was evaluated based on the appearance of newly formed bands and the disappearance of existing bands.

Homology modeling. Homology models of CARMA1 CARD and BCL10 CARD were constructed using a homology modeling server, SWISS-MODEL (22). The previously solved Apaf-1 CARD structure (PDB ID: 1CWW) and RAIDD CARD structure (PDB ID: 3CRD) were used as modeling templates for CARMA1 CARD and BCL-10 CARD, respectively. The stereochemical quality of constructed models was validated with a Ramachandran plot generated using PROCHECK (23).

Electrostatic surfaces and ribbon diagrams were generated using the PyMOL program [DeDeLano WL (2002), The 
A

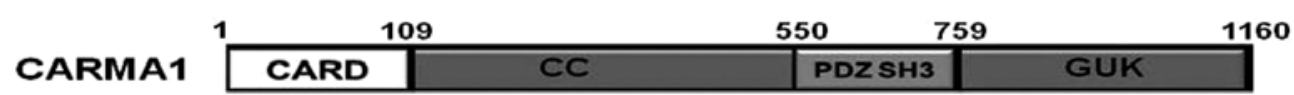

BCL10 10.233

Malt1 \begin{tabular}{c|c|c||c|c|c|c|c|}
132 & & 139 & 203 & 306 & 356 & 570 \\
\hline
\end{tabular}

B

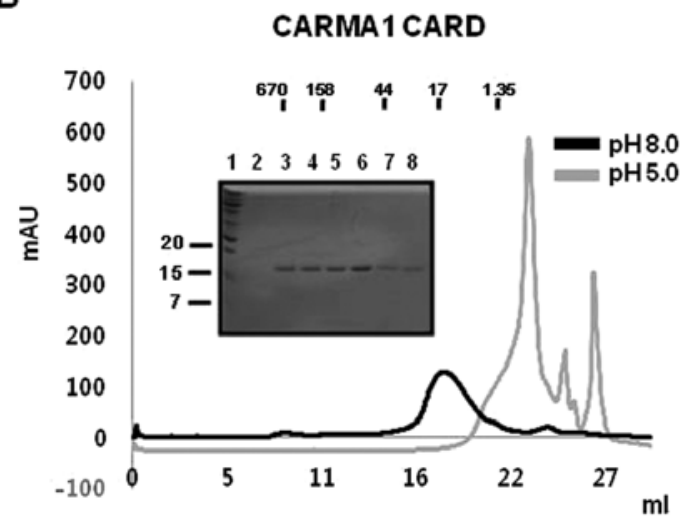

C

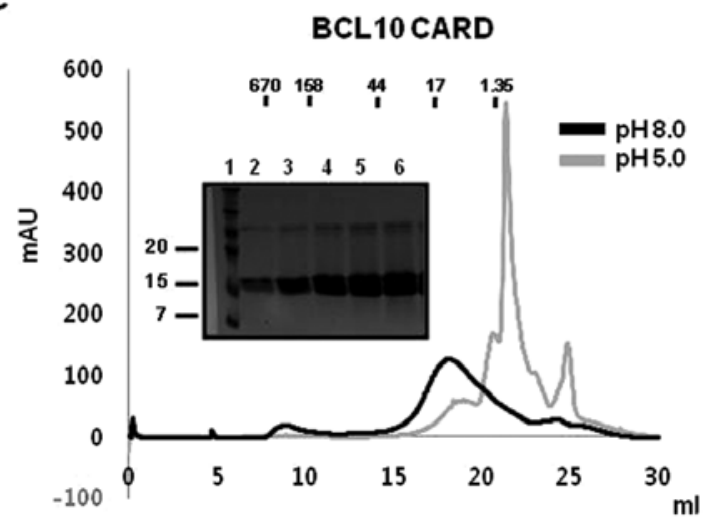

Figure 1. Purification of CARMA1 CARD and BCL10 CARD. (A) Domain boundary of protein components for the CARMA1 signalosome, CARMA1, BCL10, and MALT1. Each domain is indicated by different colors. The number of residues of amino acids corresponding to the various domains are indicated. CARD, caspase recruitment domain; CC, coiled coil domain; PDZ, Psd95, Dlg1, Zo1 domain; SH3, SRC homology 3 domain; GUK, guanylate kinase domain; DD, death domain; Ig, immunoglobulin domain. (B) Purification of CARMA1 CARD shown by gel-filtration chromatograms and fractions. The black line reflects the profile obtained under $150 \mathrm{mM} \mathrm{NaCl}$ using Tris buffer at $\mathrm{pH}$ 8.0. The gray line shows the profile obtained under $150 \mathrm{mM} \mathrm{NaCl}$ using citrate buffer at $\mathrm{pH}$ 5.0. SDS-PAGE stained by Coomassie Blue from the fractions of gel-filtration chromatography at $150 \mathrm{mM} \mathrm{NaCl}$ and $20 \mathrm{mM} \mathrm{Tris} \mathrm{buffer} \mathrm{pH} 8.0$ is shown at the upper left. Lane 1, marker; lanes 2-8, gel-filtration chromatography fractions collected from 16 to 21 ml. (C) Purification of BCL10 CARD by gel-filtration chromatograms and fractions. The black line shows the profile obtained under $150 \mathrm{mM} \mathrm{NaCl}$ using Tris buffer at $\mathrm{pH} 8.0$. The gray line shows the profile obtained under $150 \mathrm{mM} \mathrm{NaCl}$ using citrate buffer at $\mathrm{pH}$ 5.0. SDS-PAGE stained by Coomassie Blue from the fractions of gel-filtration chromatography at $150 \mathrm{mM} \mathrm{NaCl}$ and $20 \mathrm{mM}$ Tris buffer $\mathrm{pH} 8.0$ is shown on the upper left. Lane 1, marker; lanes 2-6, gel-filtration chromatography fractions collected from 15 to $20 \mathrm{ml}$.

PyMOL Molecular Graphics System, DeLano Scientific, San Carlos, CA, USA].

\section{Results}

Expression, purification and characterization of CARMAI CARD and BCL10 CARD. CARMA1, BCL10, and MALT1 proteins are the main components of the CARMA1 signalosome, which plays a critical role in lymphocyte activation and proliferation through immune cell-mediated NF- $\kappa$ B activation. Each protein contains one member of the DD superfamily, CARD for CARMA1 and BCL10, and DD for MALT1 at the $\mathrm{N}$-terminus to enable homotypic protein-protein interaction (Fig. 1A).

As a first step for elucidation of the molecular basis of assembly of the CARMA1 signalosome, which is mediated by the well known DD superfamily protein interaction module, we attempted to express and purify CARMA1 CARD and BCL10 CARD. Although it is well known that obtaining functional DD superfamily members is difficult due to their insolubility under physiological conditions (17-19), following IPTG induction bacterial cells (BL21-DE3 strain) containing CARMA1
CARD (1-109) in pET28a and BCL10 CARD (1-93 residue) in pET26b were found to express recombinant proteins with molecular weights $\sim 12$ and $10 \mathrm{kDa}$, respectively, that were detected in the soluble fraction after sonication (Fig. 1B). Both proteins were purified by $\mathrm{Ni}$ affinity chromatography followed by gel-filtration chromatography and eluted at $\sim 17 \mathrm{ml}$ of gelfiltration column, indicating they mainly exist as monomers in solution (Fig. 1B and C). Based on previous studies showing that the solubility of many DD superfamily members is sensitive to $\mathrm{pH}$ and salt concentration (20,24-26), we analyzed the characteristics of each CARD domain of CARMA1 and BCL10. Our gel-filtration chromatography study showed that both CARDs were well behaved at $\mathrm{pH} 8.0$ and not stable at pH 5.0 (Fig. 1B and C). Although both CARDs exhibited different characteristics without any salt, the salt concentration did not have a great effect on the behavior of CARDs in solution (Fig. 2A and B).

Structures of purified CARMA1 CARD and BCL1O CARD are typical $\alpha$-helix bundle folds. To confirm the correct folding and analyze the secondary structures of CARMA1 CARD and BCL10 CARD, the $\alpha$-helices were analyzed by measuring 
A

CARMA1CARD

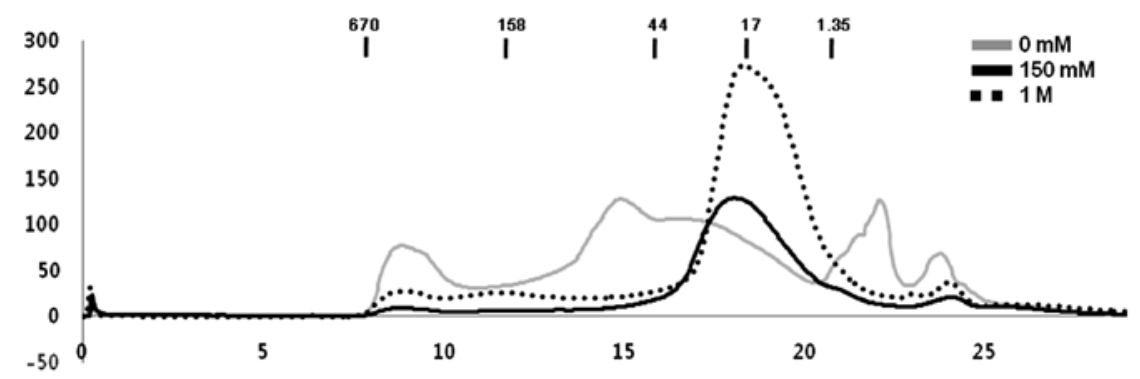

B BCL10 CARD

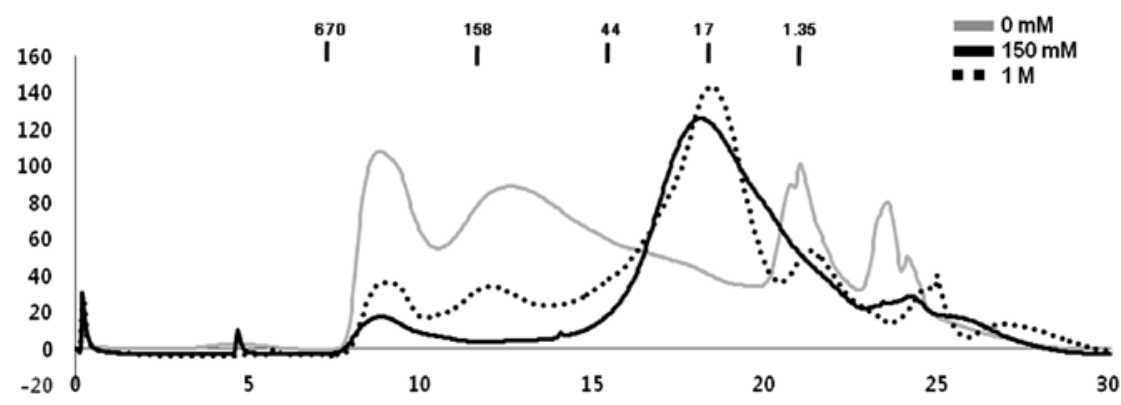

Figure 2. Effect of salt concentration on the solubility of CARMA1 CARD and BCL10 CARD. (A) Gel-filtration chromatography profile of CARMA1 CARD obtained under $0 \mathrm{mM}, 150 \mathrm{mM}$ and $1 \mathrm{M} \mathrm{NaCl}$ conditions is indicated by the gray line, black line and black dots, respectively. (B) Gel-filtration chromatography profile of BCL10 CARD obtained under $0 \mathrm{mM}, 150 \mathrm{mM}$ and $1 \mathrm{M} \mathrm{NaCl}$ conditions, is indicated by the gray line, black line and black dots, respectively.

A

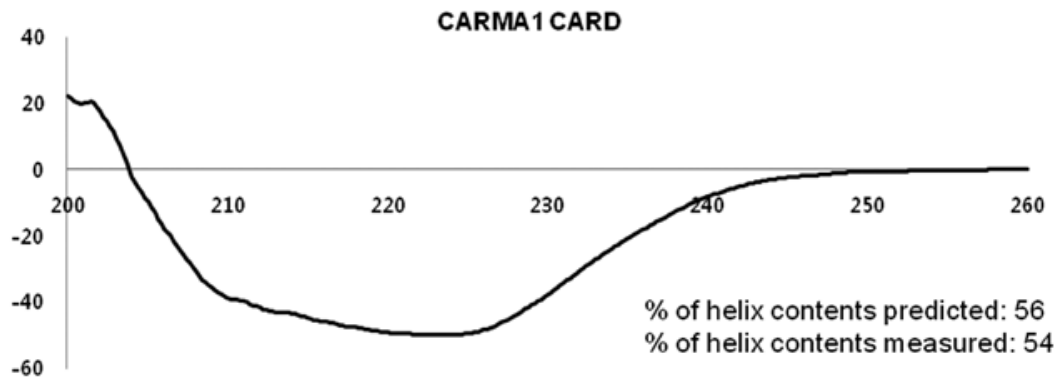

B

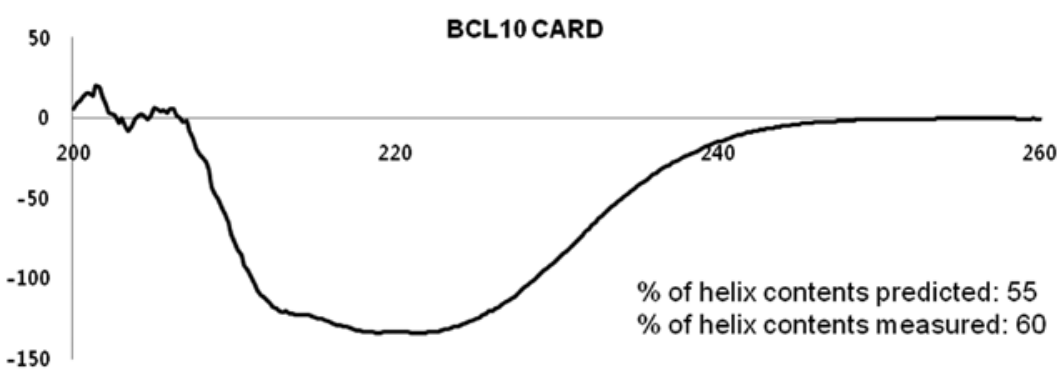

Figure 3. Circular dichroic spectra of purified (A) CARMA1 CARD and (B) BCL10 CARD. The spectra were recorded at $25^{\circ} \mathrm{C}$, and 4 scans were conducted and averaged using a J-715 spectropolarimeter. The percentage of helix contents predicted was calculated using the GOR IV secondary structure prediction server (http://npsa-pbil.ibcp.fr/cgi-bin/npsa automat.pl?page=npsa gor4.html).

the far UV circular dichroic spectra. As shown in Fig. 3, both domains showed CD spectrum patterns typical of $\alpha$-helical proteins, exhibiting two pronounced minima at $208 \mathrm{~nm}$ and $222 \mathrm{~nm}$, and a maxima at $195 \mathrm{~nm}$, which matched well with the molecular structure of other members of the death domain superfamily (20). The percentage of helix contents predicted and measured agreed well, with $56 \%$ predicted vs. $54 \%$ measured for CARMA1 and 55\% predicted vs. $60 \%$ measured for BCL10.

Purified CARMAI CARD and BCL10 CARD did not interact with each other in vitro. Since it is well known that CARMA1 
A

\section{Tris pH 8.0 and $0 \mathrm{M} \mathrm{NaCl}$}

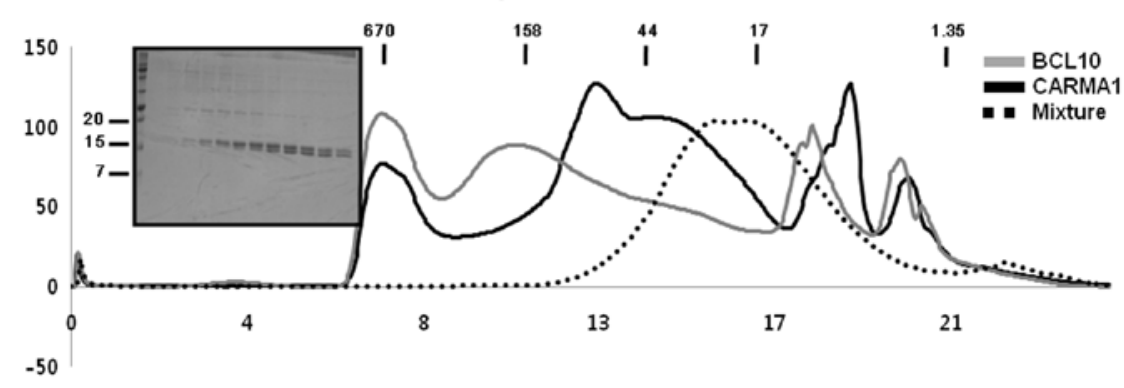

B

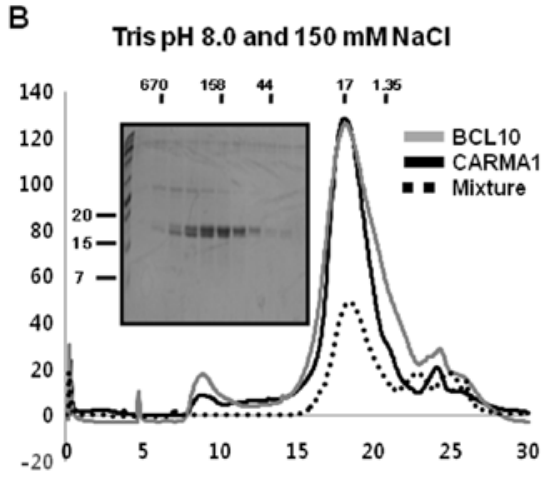

C

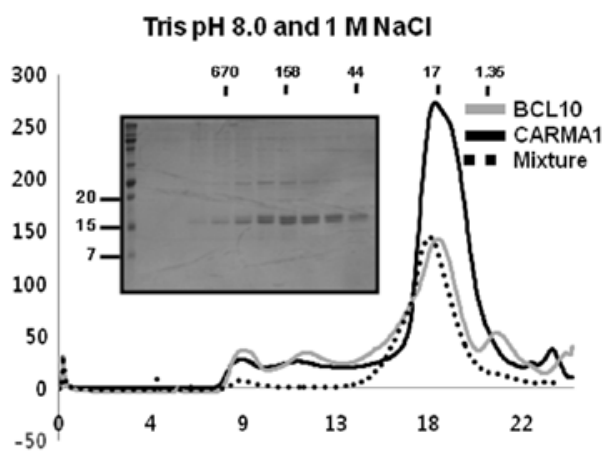

Figure 4. Complex formation assay by gel-filtration chromatography. Separately purified CARMA1 CARD and BCL10 CARD were mixed together, incubated at room temperature for $1 \mathrm{~h}$ and then loaded onto a gel-filtration column that had been pre-equilibrated with buffer containing (A) $20 \mathrm{mM}$ Tris- $\mathrm{HCl} \mathrm{pH} 8.0$ and $0 \mathrm{M} \mathrm{NaCl}$, (B) $20 \mathrm{mM}$ Tris- $\mathrm{HCl}$ pH 8.0 and $150 \mathrm{mM} \mathrm{NaCl}$ and (C) $20 \mathrm{mM}$ Tris- $\mathrm{HCl} \mathrm{pH} 8.0$ and $1 \mathrm{M}$ NaCl. SDS-PAGE of gel filtration fractions obtained from the mixture are shown at the left of the peak.

CARD can interact with BCL10 CARD directly for the assembly of the CARMA1 signalosome $(27,28)$, we tested whether purified CARMA1 CARD can interact with purified BCL10 CARD in vitro. To accomplish this, purified CARMA1 CARD was incubated with purified BCL10 CARD for $1 \mathrm{~h}$ at room temperature, after which the mixture was applied to a Superdex 200 gel-filtration column 10/30 (GE Healthcare) that had been pre-equilibrated with the proper solution. Assembly of the complex was evaluated based on the positions of the eluted peak monitored at $280 \mathrm{~nm}$ followed by SDS-PAGE. Since the interaction of proteins is usually affected by the concentration of salt, we tested the interaction between CARMA1 CARD and BCL10 CARD under no salt, low salt $(150 \mathrm{mM} \mathrm{NaCl})$ and high salt $(1 \mathrm{M} \mathrm{NaCl})$ conditions. Because the individual components and the protein mixture were both eluted at $\sim 17-18 \mathrm{ml}$ without any possible new complex peak, we concluded that no complex of CARMA1 CARD and BCL10 CARD was obtained, regardless of how much salt was added to the solution (Fig. 4A-C). Although there was a new peak generated by the protein mixture when we used the no-salt buffer during gel filtration, SDS-PAGE showed that CARMA1 CARD and BCL10 CARD did not co-migrate, indicating that they had not formed a complex (Fig. 4A).

Self-oligomerization of CARD domain prevents complex formation in the CARMAl signalosome. Formation of a complex between CARMA1 CARD and BCL10 CARD was also assayed by native-(non-denaturing) PAGE. Separately purified CARMA1 CARD and BCL10 CARD were incubated for $1 \mathrm{~h}$ at room temperature, after which the mixture was applied to the native gel $(8-25 \%$ gradient gel from
GE Healthcare). The assembly of the complex was then evaluated based on the appearance of newly formed bands and the disappearance of existing bands. No marked changes in band pattern were detected upon native-PAGE, indicating that purified CARMA1 CARD cannot form a stable complex with BCL10 CARD (Fig. 5A). Of note, although CARMA1 CARD and BCL10 CARD were detected as a single band upon SDS-PAGE (Fig. 5B), they occurred as many bands upon native-PAGE, indicating that CARMA1 CARD and BCL10 CARD easily form a highly oligomeric form, and that this self-oligomerization prevents complex formation between CARMA1 CARD and BCL10 CARD.

Predicted model of the interaction between CARMAI CARD and BCL10 CARD. To determine whether the interaction between CARMA1 CARD and BCL10 CARD for assembly of the CARMA1 signalosome occurs via a similar mode of interactions that were detected in the Apaf-1 CARD:caspase-9 CARD complex for assembly of the apoptosome, we conducted homology modeling using the Apaf-1 CARD structure (PDB ID: 1CWW) for CARMA1 CARD and the RAIDD CARD structure (PDB ID: 3CRD) for BCL10 CARD as the modeling template. Apaf-1 CARD shares $20 \%$ amino acid sequence identity with CARMA1 CARD and is the most structurally similar CARD to CARMA1 CARD (Fig. 6A). RAIDD CARD shows $21 \%$ amino acid sequence identity with BCL10 CARD (Fig. 6B). The modeled structures of CARMA1 CARD (Fig. 6C) and BCL10 CARD (Fig. 6D) were well constructed, possessing 6 helix bundles, which is the typical structural composition of the DD superfamily. To analyze the possible interaction interface, the modeling 
A

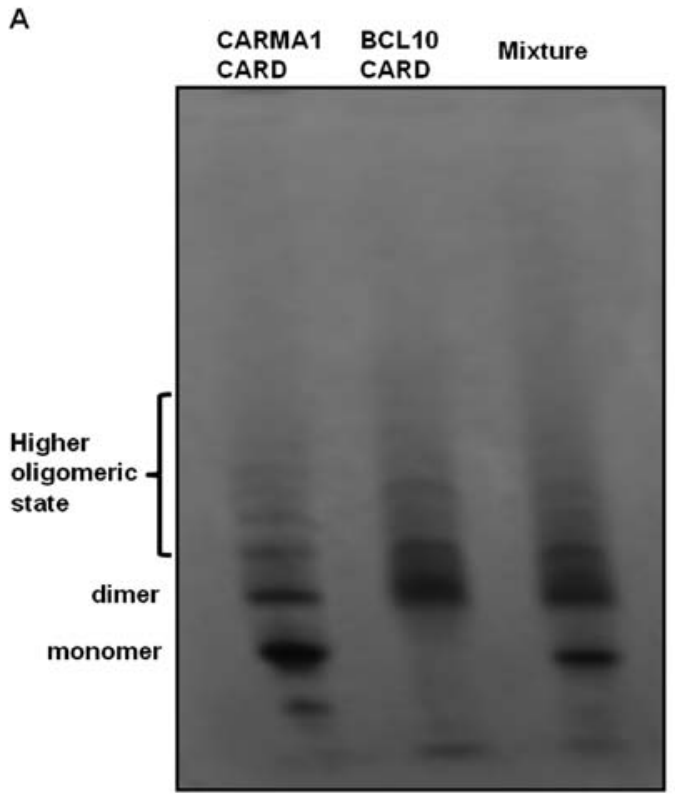

B

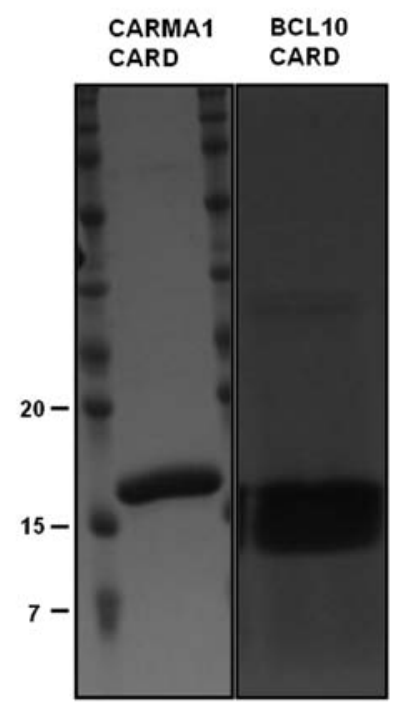

Figure 5. Self-oligomerization of CARDs detected by native-PAGE assay. (A) Native-PAGE analysis of the specific interactions between CARMA1 CARD and BCL10 CARD. (B) SDS-PAGE of purified CARMA1 CARD and BCL10 CARD.

A

CARMA1 14 TLKDEEEALWDNVECNRHMLSRYINPAKLTPYLRQCKVIDEQDEDEVLNAPMLPSKINRA 73

Apaf-1 2 PLGSMDAKARNCLLQHREALEKDIKTSYIMDHMISDGFLTISEEEKVRN- - -EPTQQQRA 58

CARMA1 74 GRLLDILHTKGQRGYVVFLESLEFY-YPELYKLVTGK 109

Apaf-1 59 AMLIKMILKKDNDSYVSFYNALLHEGYKDLAALLHDG 95

B
BCL10 13 LTEVKKDALENLRVYLCEKIIA-ERHFDHLRAKKILSREDTEEISCRTSSRKRAGKLLDY 71
RAIDD 1 MEARDKQVLRSLRLELGAEVLVEGLVLQYLYQEGILTENHIQE INAQTTGLRKTMLLLDI 60
BCL10 72 L-QENPRGLDTLVESIRREKTQSFLIQKITDEVLKLRNI 109
RAIDD 61 LPSRGPKAFDTFLDSLQE---FPWVREKLKKAREEAMTD 96

C

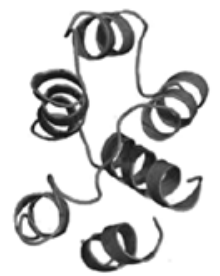

D

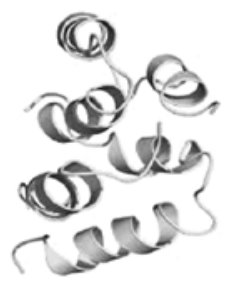

E

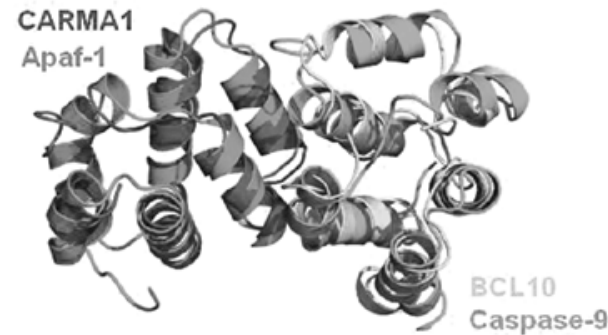

Figure 6. Homology modeling of the structures of CARMA1 CARD and BCL10 CARD. (A) Sequence alignment between CARMA1 CARD and Apaf-1 CARD. Apaf-1 CARD was the most structurally similar to CARMA1 CARD (20\% amino acid homology) and was used as a template for homology modeling of the structure of CARMA1 CARD. (B) Sequence alignment of BCL10 CARD and RAIDD CARD. RAIDD CARD was the most structurally similar to BCL10 CARD (21\% amino acid sequence homology) and was used as a template for homology modeling of the structure of BCL10 CARD. (C) Model of the structure of CARMA1 CARD predicted by SWISS-MODEL based on the template structure of Apaf-1 CARD (PDB ID: 1CWW). (D) Model of the structure of BCL10 CARD predicted by SWISS-MODEL based on the template structure of RAIDD CARD (PDB ID: 3CRD). (E) Superimposed image of CARMA1 CARD and BCL10 CARD with the Apaf-1 CARD:caspase-9 CARD complex. CARMA1 CARD and BCL10 CARD were superimposed with Apaf-1 CARD and caspase-9 CARD, respectively.

structure of CARMA1 CARD and BCL10 CARD was superimposed with the previously solved Apaf-1 CARD:caspase-9 CARD complex structure, which is the only available complex structure of the CARD:CARD interaction (Fig. 6E). Based on the structure of the Apaf-1 CARD:caspase-9 CARD complex, the interaction is mainly mediated by charged interactions. Three positively charged residues in caspase-9 CARD, R13, R52 and R56, and two negatively charged residues in Apaf-1 CARD, D27 and E40, are critical to this interaction. Sequence alignment revealed that all charged residues that are critical to 
A

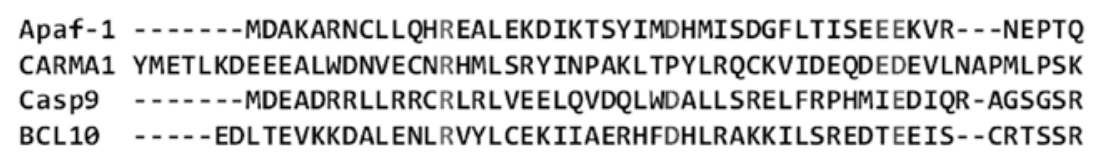

Apaf-1 QQRAAMLIKMILKKDNDSYVSFYNALLHEGYKDLAALLHDG - -

CARMA1 INRAGRLLDILHTKGQRGYVVFLESLEFYYPELYKLVTGKE - - - - - -

Casp9 RDQARQLIIDLETRGSQALPLF ISCLEDTGQDMLASFLRTN - . - . .

BCL10 KRAGK-LLDYLQEN-PRGLDTLVESIRREKTQSFLIQKITDEVLKLRNIK

B
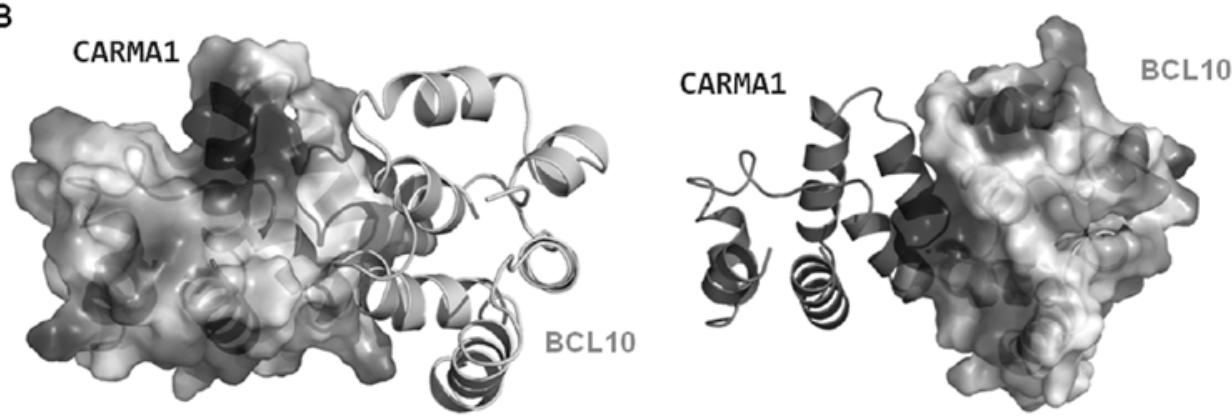

Figure 7. Model of the interaction between CARMA1 CARD and BCL10 CARD. (A) Sequence alignment between CARDs. (B) Model of interaction between CARMA1 CARD and BCL10 CARD.

the interaction between Apaf-1 CARD and caspase-9 CARD are conserved at CARMA1 CARD (E56) and BCL10 CARD (R25, K63 and K67) (Fig. 7A). The gross features of each electrostatic surface of CARMA1 CARD and BCL10 CARD were very similar to those of Apaf-1 CARD and caspase-9 CARD in that one side of CARD is acidic and the other side is basic (Fig. 7B).

\section{Discussion}

The CARMA1 signalosome, which is composed of CARMA1, BCL10 and MALT1, is a signaling molecular complex that plays a pivotal role in T-cell receptor (TCR)- and B-cell receptor (BCR)-mediated NF- $\mathrm{KB}$ activation. Each protein contains one member of the death domain (DD) superfamily, CARD for CARMA1 and BCL10 and DD for MALT1, to enable homotypic protein-protein interaction. As a first step in elucidation of the molecular basis for assembly of the CARMA1 signalosome, we attempted to express and purify CARMA1 CARD and BCL10 CARD and to reconstitute the complex in vitro for further structural studies. Since it is well known that obtaining members of the functional DD superfamily including CARD is difficult due to their insolubility under physiological conditions and the sensitivity of many DD superfamily members to $\mathrm{pH}$ and salt concentration, we analyzed the characteristics of each CARD domain of CARMA1 and BCL10 to identify the optimal conditions for purification and reconstitution.

The solubility of both CARDs was best at $\mathrm{pH} 8.0$ and between $150 \mathrm{mM}$ and $1 \mathrm{M} \mathrm{NaCl}$. CARMA1 CARD and BCL10 CARD contain an $\alpha$-helix, which match well with the molecular structure of other members of the death domain superfamily. Since it is well known that CARMA1 can interact with BCL10 directly via CARD for assembly of the CARMA1 signalosome in the cell, we tested whether purified CARMA1 CARD could interact with purified BCL10 CARD in vitro and found that separately purified CARDs did not form a stable complex. Based on the native-PAGE study, we determined that CARDs did not interact with each other as CARMA1 CARD easily self-oligomerized under certain conditions, preventing interaction with BCL10 CARD, which is a proposed partner for interaction in the cell.

Finally, the possible interaction mode between CARMA1 and BCL10 for assembly of the CARMA1 signalosome was proposed by sequence analysis and a modeling study. Comparison of the amino acid sequences and the structure of the Apaf-1 CARD:caspase-9 CARD complex (the only available CARD complex structure) revealing three positively charged residues at BCL10 CARD (R25, K63 and K67), which is well conserved in caspase-9 CARD (R13, R52 and R56), and one negatively charged residue at CARMA1 CARD (E56), which is well conserved in Apaf-1 CARD (E40), may be crucial for the interaction. Since the CARD domains are protein interaction modules, their surface features dictate their mode of interactions with partners. In the case of CARMA1 CARD and BCL10 CARD interaction for assembly of the CARMA1 signalosome, although no structural information is available, it is possible that its charged surface is important to the interaction. The DD, which is in the same superfamily as CARD, forms a highly oligomeric complex as well as a dimeric complex $(29,30)$. Although only one dimeric complex has been reported for CARD, DED and PYD, to date, it is still possible that other subfamily members of the DD superfamily including CARD, DED and PYD form more complicated oligomeric complexes similar to DD. The advantages and disadvantages of forming highly oligomeric complexes to transmit signals via the DD superfamily should be investigated in detail in future studies. 


\section{Acknowledgements}

This research was supported by the National Research Foundation of Korea (NRF) grant funded by the Korean Government (MEST) (2012-010870).

\section{References}

1. Li Q and Verma IM: NF-kappaB regulation in the immune system. Nat Rev Immunol 2: 725-734, 2002.

2. Thome M: CARMA1, BCL-10 and MALT1 in lymphocyte development and activation. Nat Rev Immunol 4: 348-359, 2004

3. Bertin J, Wang L, Guo Y, et al: CARD11 and CARD14 are novel caspase recruitment domain (CARD)/membrane-associated guanylate kinase (MAGUK) family members that interact with BCL10 and activate NF-kappa B. J Biol Chem 276: 11877-11882, 2001.

4. Gaide O, Martinon F, Micheau O, Bonnet D, Thome M and Tschopp J: Carma1, a CARD-containing binding partner of $\mathrm{Bcl10}$, induces Bcl10 phosphorylation and NF-kappaB activation. FEBS Lett 496: 121-127, 2001

5. Park HH, Lo YC, Lin SC, Wang L, Yang JK and Wu H: The death domain superfamily in intracellular signaling of apoptosis and inflammation. Annu Rev Immunol 25: 561-586, 2007.

6. Weber $\mathrm{CH}$ and Vincenz C: The death domain superfamily: a tale of two interfaces? Trends Biochem Sci 26: 475-481, 2001.

7. Reed JC, Doctor KS and Godzik A: The domains of apoptosis: a genomics perspective. Sci STKE 2004: re9, 2004.

8. Park HH, Logette E, Rauser S, et al: Death domain assembly mechanism revealed by crystal structure of the oligomeric PIDDosome core complex. Cell 128: 533-546, 2007.

9. Zhou H, Wertz I, O'Rourke K, et al: Bcl10 activates the NF-kappaB pathway through ubiquitination of NEMO. Nature 427: 167-171, 2004

10. Oeckinghaus A, Wegener E, Welteke V, et al: Malt1 ubiquitination triggers NF-kappaB signaling upon T-cell activation. EMBO J 26: 4634-4645, 2007.

11. Rebeaud F, Hailfinger S, Posevitz-Fejfar A, et al: The proteolytic activity of the paracaspase MALT1 is key in T cell activation. Nat Immunol 9: 272-281, 2008.

12. Coornaert B, Baens M, Heyninck K, et al: $\mathrm{T}$ cell antigen receptor stimulation induces MALT1 paracaspase-mediated cleavage of the NF-kappaB inhibitor A20. Nat Immunol 9: 263-271, 2008.

13. Uren AG, O'Rourke K, Aravind LA, et al: Identification of paracaspases and metacaspases: two ancient families of caspase-like proteins, one of which plays a key role in MALT lymphoma. Mol Cell 6: 961-967, 2000

14. Lucas PC, Yonezumi M, Inohara N, et al: Bcl10 and MALT1, independent targets of chromosomal translocation in malt lymphoma, cooperate in a novel NF-kappa B signaling pathway. J Biol Chem 276: 19012-19019, 2001.
15. Sun Z, Arendt CW, Ellmeier W, et al: PKC-theta is required for TCR-induced NF-kappaB activation in mature but not immature T lymphocytes. Nature 404: 402-407, 2000.

16. Matsumoto R, Wang D, Blonska $\mathrm{M}$, et al: Phosphorylation of CARMA1 plays a critical role in T cell receptor-mediated NF-kappaB activation. Immunity 23: 575-585, 2005.

17. Huang B, Eberstadt M, Olejniczak ET, Meadows RP and Fesik SW: NMR structure and mutagenesis of the Fas (APO-1/CD95) death domain. Nature 384: 638-641, 1996.

18. Jang TH and Park HH: Purification, crystallization and preliminary X-ray crystallographic studies of RAIDD Death-Domain (DD). Int J Mol Sci 10: 2501-2509, 2009.

19. Jeong EJ, Bang S, Lee TH, Park YI, Sim WS and Kim KS: The solution structure of FADD death domain. Structural basis of death domain interactions of Fas and FADD. J Biol Chem 274: 16337-16342, 1999.

20. Jang TH and Park HH: Generalized semi-refolding methods for purification of the functional death domain superfamily. J Biotechnol 151: 335-342, 2011

21. Chen YH, Yang JT and Martinez HM: Determination of the secondary structures of proteins by circular dichroism and optical rotatory dispersion. Biochemistry 11: 4120-4131, 1972.

22. Schwede T, Kopp J, Guex N and Peitsch MC: SWISS-MODEL An automated protein homology-modeling server. Nucleic Acids Res 31: 3381-3385, 2003.

23. Laskowski RA, Rullmannn JA, MacArthur MW, Kaptein R and Thornton JM: AQUA and PROCHECK-NMR: programs for checking the quality of protein structures solved by NMR. J Biomol NMR 8: 477-486, 1996.

24. Bae JY and Park HH: Crystal structure of NALP3 protein pyrin domain (PYD) and its implications in inflammasome assembly. J Biol Chem 286: 39528-39536, 2011.

25. Bae JY and Park HH: Crystallization and preliminary X-ray crystallographic studies of the PYD domain of human NALP3. Acta Crystallogr Sect F Struct Biol Cryst Commun 67: 1421-1424, 2011.

26. Park HH: Structural analyses of death domains and their interactions. Apoptosis 16: 209-220, 2011.

27. Wegener E and Krappmann D: CARD-Bcl10-Malt1 signalosomes: missing link to NF-kappaB. Sci STKE 2007: pe21, 2007.

28. Liu Y, Song R, Gao Y, et al: Protein kinase C-delta negatively regulates $\mathrm{T}$ cell receptor-induced NF-kappaB activation by inhibiting the assembly of CARMA1 signalosome. J Biol Chem 287: 20081-20087, 2012

29. Park HH: Structural features of caspase-activating complexes. Int J Mol Sci 13: 4807-4818, 2012.

30. Ferrao R and Wu H: Helical assembly in the death domain (DD) superfamily. Curr Opin Struct Biol 22: 241-247, 2012. 